\title{
Fate of the Wiedemann-Franz law near quantum critical points of electron systems in solids
}

\author{
V. A. Khodel, ${ }^{1,2}$ J. W. Clark, ${ }^{2,3}$ V. R. Shaginyan, ${ }^{4,5}$ and M. V. Zverev ${ }^{1,6}$ \\ ${ }^{1}$ NRC Kurchatov Institute, Moscow, 123182, Russia \\ ${ }^{2}$ McDonnell Center for the Space Sciences \& Department of Physics, \\ Washington University, St. Louis, MO 63130, USA \\ ${ }^{3}$ Centro de Ciências Matemáticas, Universidade de Madeira, 9000-390 Funchal, Madeira, Portugal \\ ${ }^{4}$ Petersburg Nuclear Physics Institute, NRC Kurchatov Institute, Gatchina, 188300, Russia \\ ${ }^{5}$ Clark Atlanta University, Atlanta, GA 30314, USA \\ ${ }^{6}$ Moscow Institute of Physics and Technology, Dolgoprudny, Moscow District 141700
}

\begin{abstract}
We introduce and analyze two different scenarios for violation of the Wiedemann-Franz law in strongly correlated electron systems of solids, close to a topological quantum critical point (TQCP) where the density of states $N(0)$ diverges. The first, applicable to the Fermi-liquid (FL) side of the TQCP, involves a transverse zero-sound collective mode that opens a new channel for the thermal conductivity, thereby enhancing the Lorenz number $L(0)$ relative to the value $L_{0}=\pi^{2} k_{B}^{2} / 3 e^{2}$ dictated by conventional FL theory. The second mechanism for violation of the WF law, relevant to the non-Fermi-liquid (NFL) side of the TQCP, involves the formation of a flat band and leads instead to a reduction of the Lorenz number.

PACS numbers: 71.10.Hf, 71.27.+a, 71.10.Ay
\end{abstract}

Introduction. The perplexing issue of a possible violation of the Wiedemann-Franz (WF) law in strongly correlated electron systems of solids has recently attracted much attention. The WF law states that as the temperature $T$ goes to zero, the product $L(T)=\kappa \rho / T$ of the thermal conductivity $\kappa$ and the electrical resistivity $\rho$, divided by $T$, always approaches the Lorenz number $L_{0}=\pi^{2} k_{B}^{2} / 3 e^{2}$. Over a brief period, several articles have appeared [1 [6] whose authors reported or discussed putative evidence of a breakdown of this fundamental law in the antiferromagnetic (AF) heavy-fermion metal $\mathrm{YbRh}_{2} \mathrm{Si}_{2}$. This compound has become one of the prime test cases in the quest for understanding of NFL behavior of electron systems that exhibit quantum critical points (QCPs) at which lines of second-order phase transitions are terminated. Conclusions drawn about the fate of the WF law have varied, but the authors of Ref. [1] assert that the WF law is definitely violated.

When one considers the possibility of violation of the WF law, it must be emphasized that this law is, in fact, well satisfied across the wide range of metallic electron systems that have been studied experimentally, except for two particularly interesting compounds in addition to $\mathrm{YbRh}_{2} \mathrm{Si}_{2}$. The first is the normal state of the superconducting heavy-fermion metal $\mathrm{CeCoIn}_{5}[8$ and the second, the heavy-fermion compound YbAgGe [9]. QCPs of the type indicated above are present in the phase diagrams of all three of these electron systems.

It is important to recognize that in all the cited publications claiming that the WF law is definitely or likely to be violated, the temperatures reached in the measurements involved were no lower than $40 \mathrm{mK}$. However, in recent studies of $\mathrm{YbRh}_{2} \mathrm{Si}_{2}$, measurements have been performed at considerably lower temperatures $T \simeq$
$10 \mathrm{mK}$. Most significantly, the thermal resistivity $w(T)=$ $L_{0} T / \kappa(T)$ was observed to execute an unexpected downturn at $T \leq 30 \mathrm{mK}$, shown in Fig. 1 of Refs. [4, 5]. This behavior confounds any strong conclusions previously made about the fate of the WF law in this compound and casts doubt on others.

Preliminaries: relevant phenomena in the quantum critical regime. According to the authors of Refs. [4, 5], the occurrence of the downturn in the thermal resistivity $w(T)$ points to the presence of an unknown collective mode, since all phonon contributions have disappeared already by $T \leq 1 \mathrm{~K}$. One might then suppose that in the AF state of $\mathrm{YbRh}_{2} \mathrm{Si}_{2}$, which terminates at $T_{N} \simeq 70 \mathrm{mK}$, magnons can provide the anticipated boson mode. This is not the case, however, since the downturn in $w(T)$ observed at $T \leq 30 \mathrm{mK}$ persists in external magnetic fields whose magnitude is in excess of a critical value sufficient for termination of the AF ordering [4, 5].

On the other hand, there is a special feature of the phase diagram of $\mathrm{YbRh}_{2} \mathrm{Si}_{2}$ that is relevant to a search for the posited boson mode. Namely: the AF QCP resides close to a topological quantum critical point (TQCP) associated with the divergence of the density of states $N(0)$, which in a homogeneous Fermi liquid is proportional to the effective mass $M^{*}$. The two critical points, i.e., the TQCP and that of the termination of the $\mathrm{AF}$ state at $T_{N}$, coincide in the presence of a tiny external magnetic field $B_{c} \simeq 70 \mathrm{mT}[10,11]$. That the $\mathrm{AF}$ state of $\mathrm{YbRh}_{2} \mathrm{Si}_{2}$ lies on the FL side of the TQCP is confirmed experimentally by verification that its thermodynamic and kinetic properties, such as the specific heat and resistivity, behave in accordance with the predictions of FL theory.

The proximity of a Fermi system to its TQCP cre- 
ates the opportunity for propagation of a transverse zero sound mode, henceforth referred to as a zeron for brevity, which is known to propagate in $3 \mathrm{D}$ liquid ${ }^{3} \mathrm{He}$. Applicability of the zeron scenario to elucidation of properties of $2 \mathrm{D}$ liquid ${ }^{3} \mathrm{He}$ has been explored in Refs. [12 14]. In general, the zeron mode provides an additional contribution to the thermal conductivity $\kappa$ analogous to that coming from phonons and/or magnons. However, in contrast to the magnon mode, the zeron contribution to $\kappa$ is quite insensitive to the imposition of external magnetic fields $B$, a feature consistent with experimental observations of the behavior of $\kappa(B)$ in $\mathrm{YbRh}_{2} \mathrm{Si}_{2}$ [4, 5].

A different mechanism for violation of the WF law comes into play on the NFL side of the TQCP. Beyond this point it is no longer the case that variation of the ground-state energy $E$, given by the FL expression

$$
\delta E=\sum \epsilon(\mathbf{p}) \delta n(\mathbf{p}),
$$

yields a positive result for any admissible variation of the Landau quasiparticle momentum distribution $n(\mathbf{p})$. This breakdown triggers a rearrangement of the Landau state [15]. The WF law can be profoundly affected if the rearrangement involves a specific topological phase transition traditionally known as fermion condensation, which gives rise to the formation of flat bands [16 20]. Emergence of a flat band entails renormalization of conventional FL formulas, notably those in which the residual electrical and thermal resistivities, respectively $\rho_{0}$ and $w_{0}$, are necessarily attributed to impurities. It will be shown that the impurity-independent flat-band contribution to $\rho_{0}$ is always less than that to $w_{0}$. Consequently, the net effect associated with the flat-band scenario on the WF law is a decline of the ratio $L(0) / L_{0}$ from unity, in agreement with available experimental data [2, 5, 8, ,9].

Violation of WF law: zeron scenario. We now turn to a more detailed analysis of the posited scenarios for violation of the WF law, beginning with the zeron mechanism for the recently observed downturn of the thermal resistivity. On the FL side of the TQCP, the intervention of the zeron mode can be addressed with the aid of the standard FL kinetic equation [21, 22]

$$
\left(\omega_{k}-\mathbf{k} \frac{\partial \epsilon(\mathbf{p})}{\partial \mathbf{p}}\right) \phi(\mathbf{p}, \mathbf{k})=-\mathbf{k} \frac{\partial n(\mathbf{p})}{\partial \mathbf{p}} \int f\left(\mathbf{p}, \mathbf{p}_{1}\right) \phi\left(\mathbf{p}_{1}, \mathbf{k}\right) d v_{1},
$$

in which $\phi(\mathbf{p}, \mathbf{k})$ describes the deviation of the quasiparticle momentum distribution from equilibrium, $f\left(\mathbf{p}, \mathbf{p}_{1}\right)$ is the Landau interaction function, and $d v$ is the volume element in 3D momentum space, while $\omega_{k}=c_{z} k$. The impact of an external field due to the crystal lattice will be discussed later.

By way of preparation, it is helpful first to consider the basic model of a homogeneous Fermi liquid in which a single band crosses the Fermi surface. In this case, the dispersion equation (2) for the zeron group velocity $c_{z}$ is conveniently recast as [23]

$$
1-\frac{6}{F_{1}}=3\left(\frac{c_{z}^{2}}{v_{F}^{2}}-1\right)\left(\frac{c_{z}}{2 v_{F}} \ln \frac{c_{z}+v_{F}}{c_{z}-v_{F}}-1\right)
$$

in terms of the dimensionless first harmonic $F_{1}=f_{1} N(0)$ of the Landau interaction function $f$, where $N(0)=$ $p_{F} M^{*} / \pi^{2}$ is the density of states of $3 \mathrm{D}$ homogeneous matter. Here we may locate the TQCP itself at the point where $v_{F}=p_{F} / M^{*}=0$, which is determined by the relation [21]

$$
1=\frac{1}{3} f_{1} N_{0}(0)
$$

in terms of the density of states $N_{0}(0)=p_{F} M / \pi^{2}$ of noninteracting quasiparticles. As seen from Eq. (3), a nontrivial solution does exist provided $F_{1}>6$, or equivalently $M^{*} / M>3$.

Calculations are greatly facilitated near the TQCP where $F_{1} \gg 1$; indeed they essentially coincide with those executed for longitudinal zero sound in a neutral Fermi liquid. Simple algebra leads to 23]

$$
c_{z}=\frac{v_{F}^{0}}{\sqrt{5 M^{*} / M}} \propto v_{F} \sqrt{F_{1}},
$$

where $v_{F}^{0}=p_{F} / M$. Just as for the longitudinal mode in a neutral Fermi liquid, the transverse mode in question feels no Landau damping, since $c_{z}>v_{F}$. Moreover, its dissipation at $T=0$ is also related to the generation of incoherent electron particle-hole $(p h)$ pairs. This feature allows us to adopt the same procedure in evaluating the damping $\gamma_{z}(T)$ of the zeron mode by the $e-p h$ pair mechanism, as applied to evaluation of the damping of longitudinal zero sound in neutral Fermi systems [23]. The result is $\gamma_{z}(T)=1 /\left(c_{z} \tau(T)\right)$, where $\tau(T) \propto T^{-2}$ is a time characteristic of the damping of single-particle excitations. Hence $\gamma_{z}(T \rightarrow 0) \propto T^{2}$, implying that the scattering length $l$ goes like $T^{-2}$, while the thermal conductivity $\kappa \propto C(T) l(T) v_{F}$ varies as $T^{-1}$ [25] to produce the behavior $w(T)=w_{0}+w_{2} T^{2}$.

The single-band model developed above must be modified to deal with the actual case of $\mathrm{YbRh}_{2} \mathrm{Si}_{2}$, in which several electron bands simultaneously cross the Fermi surface. Among them, those associated with light carriers of Fermi momenta $v_{l}$ play the major role. The Fermi surface of $\mathrm{YbRh}_{2} \mathrm{Si}_{2}$ is anisotropic, with heavy carriers occupying its smaller portion. In the simplest case, assumed here, the velocities of light carriers are subsumed by a single parameter $v_{l} \gg v_{F}$, and Eq. (3) is replaced by

$$
\begin{gathered}
1-\frac{6}{F_{1}}=3\left(\frac{c_{z}^{2}}{v_{F}^{2}}-1\right)\left(\frac{c_{z}}{2 v_{F}} \ln \frac{c_{z}+v_{F}}{c_{z}-v_{F}}-1\right) \\
+\frac{3 v_{F}}{v_{l}}\left(\frac{c_{z}^{2}}{v_{l}^{2}}-1\right)\left(\frac{c_{z}}{2 v_{l}} \ln \frac{c_{z}+v_{l}}{c_{z}-v_{l}}-1\right) .
\end{gathered}
$$


It is readily seen that the zeron group velocity remains almost unchanged. Indeed, upon neglecting the contribution from the light band to the real part of the right side of Eq. (6) in view of the small value of the prefactor $v_{F} / v_{l}$, we arrive at the same equation as before for the real part $c_{R}$ of the zeron group velocity $c_{z}=c_{R}+i c_{I}$. Thus, $c_{R}$ does in fact satisfy Eq. (5). A new feature of the modified model is the presence of Landau damping of the zeron mode, which comes into play because near the topological QCP, the zeron group velocity $c_{R}$ is larger than the Fermi velocity $v_{F}$ of the heavy band, while remaining less than that of the light band.

The inequality $c_{R}<v_{l}$ lifts the ban on zeron emission/absorption by electrons, since in this case the conservation law $\epsilon(\mathbf{p}+\mathbf{k})-\epsilon(\mathbf{p})-\omega_{k}=0$ is met for the light carriers, thereby opening a channel for damping of the zeron mode. Observe then that in the last term on the right side of Eq. (6) we have $\ln \left(\left(c_{z}+v_{l}\right) /\left(c_{z}-v_{l}\right)\right) \simeq$ $\ln (-1)=i \pi$, because $\left|c_{z}\right| \ll v_{l}$. This term cancels out the imaginary part of the first term to establish

$$
c_{I} \propto\left(v_{F}^{0} / v_{l}\right)^{2} \frac{p_{F}}{M^{*}} .
$$

Since $c_{I} \propto 1 / M^{*} \ll c_{R} \propto \sqrt{1 / M^{*}}$, the zeron mode turns out to be weakly damped, much like the phonon mode in solids.

Pursuing this analogy, the kinetic theory of the electron-zeron problem recapitulates that of electronphonon physics. The only significant difference naturally concerns the normalization of the electron-zeron vertex part $g$. This quantity is determined from analysis of the equation for the amplitude $\Gamma(1,2 ; \omega)$ of electron-electron scattering in the $p h$ channel near its pole $\omega=\omega_{k}$ [24]. In symbolic form one has $\Gamma=\mathcal{U}+(\mathcal{U} G G \Gamma)$, where $\mathcal{U}$ denotes the block of Feynman diagrams irreducible in the $p h$ channel and $G$ is the single-particle Green function; the brackets imply integration and summation over intermediate momentum and spin variables. This amplitude consists of a pole term $\Gamma^{P}\left(1,2, \omega \rightarrow \omega_{k}\right)=g(1) g(2) /(\omega-$ $\left.\omega_{k}\right)$ and a regular remainder $\Gamma^{R}(1,2, \omega)$ that satisfies the symbolic equation $\Gamma^{R}=\mathcal{U}+\left(\mathcal{U} G G \Gamma^{R}\right)+(\mathcal{U} X g) g$ where $X=\partial(G G) / \partial \omega$. Upon multiplying both sides of this equation by the product $g G G$ from the left and integration over intermediate momenta and summation over spins, most of the terms cancel out to yield $\left(g^{2} \partial(G G) / \partial \omega\right)=-1[24]$. In the explicit form, it reads

$$
g^{2}(k) \int \frac{(n(\mathbf{p}+\mathbf{k})-n(\mathbf{p}))(\epsilon(\mathbf{p}+\mathbf{k})-\epsilon(\mathbf{p}))}{\left(\omega_{k}^{2}-(\epsilon(\mathbf{p}+\mathbf{k})-\epsilon(\mathbf{p}))^{2}\right)^{2}} \frac{2 d^{3} p}{(2 \pi)^{3}}=\frac{1}{2 \omega_{k}} .
$$

Analysis of this relation is greatly simplified in the vicinity of the topological QCP. Since $c_{z}^{2} \gg v_{F}^{2}$, the difference $\omega_{k}^{2}-(\epsilon(\mathbf{p})+\mathbf{k})-(\epsilon(\mathbf{p}))^{2}$ reduces to its first term, $c_{z}^{2} k^{2}$; after some algebra we obtain

$$
g^{2}(k) \simeq \frac{v_{F}^{0} v_{F} \omega_{k}}{p_{F}^{3}} .
$$

The zeron contribution $\kappa_{z}(T)$ to the conventional electron thermal conductivity $\kappa_{e e}(T) \propto T^{-1}$ can be evaluated with the aid of textbook formulas. In these, the phonon Debye temperature is replaced by the zeron temperature $\Theta_{z}=c_{z} k_{\max }$, where $k_{\max }$ denotes the maximum value of the zeron wave vector. This yields (see e.g. 25])

$$
\begin{aligned}
& \kappa_{z}(T) \propto \Theta_{z}^{2} / T^{2}, \quad T<\Theta_{z}, \\
& \kappa_{z}(T)=\text { const., } \quad T>\Theta_{z} .
\end{aligned}
$$

As $T \rightarrow 0$, the zeron part of $\kappa(T)$ is seen to soar upward more rapidly than $\kappa_{e e}(T)$. Therefore $\Theta_{z}$ can in fact be responsible for the upturn of the thermal conductivity $\kappa(T)$ at $T \rightarrow 0$ reported in Refs. [4, 5], provided its value does not exceed $30 \mathrm{mK}$.

The zeron-electron collision integral $I_{z e}(T)$ is markedly enhanced in the classical-like region $T \geq \Theta_{z}$ where all the zerons have energies lower than $T$. Setting $\epsilon^{\prime}=\epsilon(\mathbf{p}+\mathbf{k})$, this integral takes the form [25]

$$
I_{e z} \propto \frac{\partial n_{0}(\epsilon)}{\partial \epsilon} \int g^{2}(k) \frac{\partial N_{z}(\omega)}{\partial \omega} \delta\left(\epsilon-\epsilon^{\prime}\right)\left(\varphi-\varphi^{\prime}\right) \frac{\omega d^{3} k}{(2 \pi)^{3}},
$$

where $\varphi(\epsilon)$ and $\varphi^{\prime}=\varphi\left(\epsilon^{\prime}\right)$ are the deviations of the electron distribution function from its equilibrium form $n_{0}(\epsilon)=(1+\exp (\epsilon / T))^{-1}$, and $g$ is again the electronzeron vertex part. The equilibrium zeron distribution function $N_{z}(\omega)$ is simply $T / \omega$. Insertion of Eq. (9) into Eq. (11) and straightforward algebraic steps lead to

$$
I_{e z}\left(T \geq \Theta_{z}\right) \simeq \frac{k_{\max }^{2}}{p_{F}^{2}} \varphi .
$$

At $T \geq \Theta_{z}$, both the electron-zeron collision integral and the zeron contribution to the thermal and electrical resistivities differ from their electron-phonon counterparts [25] by the ratio $k_{\max }^{2} / p_{F}^{2}$.

The magnitude of the factor $k_{\max }^{2} / p_{F}^{2}$ can be estimated by tracing the evolution of the spectrum $\omega_{z}(k)$ as a function of wave vector $k$. The key point is that the sign of the difference $\omega_{k}^{2}-(\epsilon(\mathbf{p}+\mathbf{k})-\epsilon(\mathbf{p}))^{2}$ in Eq. (8) must be positive. Hence $\omega_{k}$ grows rapidly as $\epsilon(\mathbf{p}+\mathbf{k})-\epsilon(\mathbf{p})$ increases, becoming a nonlinear function of $k$ in the momentum region where the spectrum $\epsilon(\mathbf{p})$ ceases to be relatively flat. Such a nonlinear behavior of $\omega_{k}$ triggers the decay of the zeron mode at a critical wave vector $k_{c r} \simeq k_{\max }$ where the relation $\omega\left(k_{c r}\right)=\omega_{k_{1}}+\omega_{k_{2}}$, with $k_{1}+k_{2}=k_{c r}$, is met for the first time, implying that the zeron mode becomes unstable at $k>k_{c r}$.

Working within the zeron scenario, we now seek a resolution of the puzzling low- $T$ behavior of the thermopower (or Seebeck coefficient) of $\mathrm{YbRh}_{2} \mathrm{Si}_{2}$, as revealed in Ref. [26]. According to conventional (i.e., FL) theory, the Seebeck coefficient $S(T)$ must vary linearly with temperature as $T \rightarrow 0$, i.e., $-S(T)=s_{F L} T$, with a positive constant $s_{F L}$ 25, 27, 28]. Instead, as measured in this compound, the function $-S(T)$ exhibits a deep 
downturn with a subsequent sign change that occurs in the same temperature region where the abrupt upturn in thermal conductivity was observed [4, 5].

This coincidence suggests that the downturn is due to zeron drag, in analogy with the well-known phonon drag. Since input parameters specifying the boson mode, such as the group velocity $c$ or the critical wave vector $k_{\max }$, are absorbed into the corresponding Debye temperature, the zeron contribution $S_{z}$ to the Seebeck $S(T)$ may be written as 25, 27]

$$
\begin{array}{r}
S_{z} \propto T^{2} / \Theta_{z}^{2}, \quad T<\Theta_{z}, \\
S_{z} \propto \Theta_{z} / T, \quad T>\Theta_{z} .
\end{array}
$$

In case the drag contribution to $S(T)$ and the standard FL contribution have opposite signs, they may cancel each other, thereby allowing the total Seebeck coefficient $S$ to change sign. The maximum $S_{z}^{\max }$ of the zeron drag term, although small, is nevertheless temperature independent 25], while the FL term $-s_{F L} T$ vanishes at $T \rightarrow 0$. It then follows that the total Seebeck coefficient $S$ becomes positive in some temperature region, provided $\Theta_{z}$ meets the requirement

$$
\Theta_{z}<S_{z}^{\max } / s_{F L} .
$$

Since the FL term in $S$ prevails at $T \rightarrow 0$, this implies that at extremely low $\Theta_{z}$, the Seebeck coefficient $S(T)$ must change sign twice, (see Fig. 1). This behavior provides a fingerprint of the zeron scenario for the NFL component of the thermopower.

The role of both these mechanisms becomes more profound in the presence of a periodic external field of the crystal lattice, with Bloch-Wannier wave functions as eigenfunctions of the solid-state Hamiltonian. As an illustration, consider the well-known tight-binding model, in which the Coulomb interaction between moving electrons and localized atoms is taken into account, while interactions between electrons themselves are neglected. The single-particle spectrum of the simplest version of this model, in which only nearest-neighbor matrix elements of the electron-atom interaction Hamiltonian are included, has the form $\epsilon(\mathbf{p}) \propto \cos p_{x} a+\cos p_{y} a+\cos p_{z} a$, where $a$ is the lattice constant. This formula implies that the inverse tight-binding group velocity $1 /|\partial \epsilon(\mathbf{p}) / \partial \mathbf{p}|$, which enters the density of states $N_{a}(0)$ of noninteracting electrons moving in the external field of the crystal lattice, diverges near the saddle points $(0, \pi) \ldots(\pi, 0)$ as $1 /\left|\sin p_{x}^{v H} a\right| \simeq 1 /\left|\sin p_{y}^{v H} a\right| \simeq 1 /\left|\sin p_{z}^{v H} a\right|$, where $\mathbf{p}^{v H}=\left(p_{x}^{v H}, p_{y}^{v H}, p_{z}^{v H}\right)$ stands for the coordinates of the van Hove point. Since the compounds of interest here have open Fermi surfaces crossing the Brillouin zone close to the saddle points, such an enhancement is often substantial.

In dealing with strongly correlated electron systems, the product $f_{1} N_{a}(0)$ must then be of order of unity (cf. Eq. (4)), assuming $f_{1}$ is again identified with the

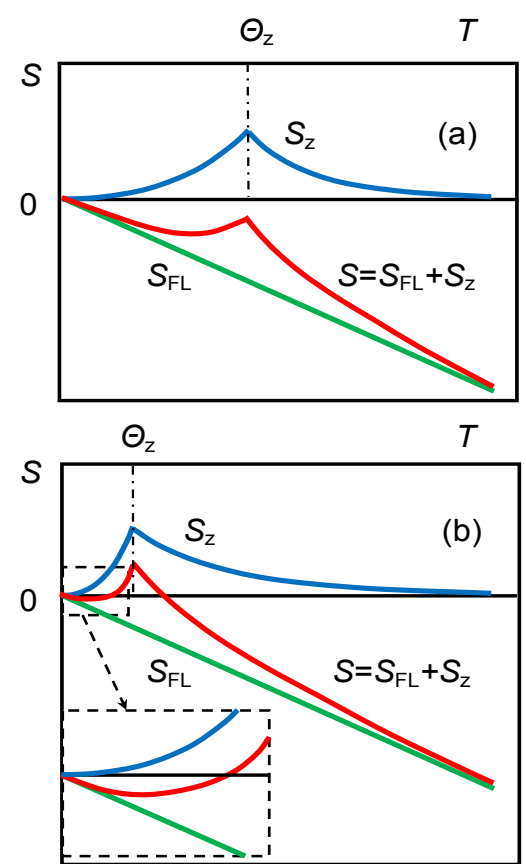

FIG. 1: Schematic illustration of the total Seebeck coefficient $S$ (red line) and its separate Fermi-Liquid $S_{F L}=-s_{F L} T$ (green line) and zeron $S_{z}$ (blue lines) contributions. In the case $\Theta_{z}>S_{z}^{\max } / s_{F L}$ (panel (a)) the total $S(T)=-s_{F L} T+$ $S_{z}(T)$ does not change sign, while in the opposite case $\Theta_{z}<$ $S_{z}^{\max } / s_{F L}$ (panel (b)) it changes sign twice.

corresponding matrix element of the effective $e-e$ interaction. Consequently, when estimating the suppression of the group velocity $c_{z}$, one can employ a modified version

$$
c_{z}=\frac{v_{F}^{0}}{\sqrt{5 M^{*} M_{a}}}
$$

of Eq. (5), in which the parameter $M_{a}$ specifies the density of states $N_{a}(0)$ in the same way as $M$ specifies the density of states $N_{0}(0)$.

The enhancement of the density of states $N(0)$ of $\mathrm{YbRh}_{2} \mathrm{Si}_{2}$ beyond what is typical of conventional metals can be estimated from experimental data on the specificheat coefficient $\gamma=C(T) / T=1.5 \mathrm{~J} /$ mole $K^{2}$. In conventional metals, the $e-e$ interaction is rather small, and the densities of states of these systems, varying in the interval $1 \div 5 \mathrm{~mJ} /$ mole $K^{2}$, are well approximated by the FL formula $N_{0}(0) \propto M$. We thus infer that the total enhancement factor $N(0) / N_{0}(0)=M^{*} / M$ for $\mathrm{YbRh}_{2} \mathrm{Si}_{2}$ is about $10^{3}$. Presumably the difference between $M^{*}$ and $M_{a}^{*}$ is not as large as that between $M^{*}$ and $M$. If so, the ratio $c_{z} / v_{F}^{0}$ is suppressed by a factor $\simeq 10^{3}$.

The impact of anisotropy effects on the magnitude of the ratio $k_{\max }^{2} / p_{F}^{2}$ is likewise well pronounced, since $\mathrm{YbRh}_{2} \mathrm{Si}_{2}$ has a typical open Fermi surface, with flattening of the single-particle spectrum occurring solely in nar- 
row regions close to the van Hove points. This situation provides for an additional decline of the characteristic zeron temperature $\Theta_{z}$, down to values compatible with the $30 \mathrm{mK}$ needed to explain the upturn of the thermal conductivity of this compound [4, 5].

Judging from the preceding theoretical development, analysis, and results, the zeron scenario for explanation of the experimental findings of Refs. [4, 5] seems to be rather plausible. If this scenario is indeed applicable, the fate of the WF law in $\mathrm{YbRh}_{2} \mathrm{Si}_{2}$ at the lowest accessible temperatures can be clarified only by subtracting the zeron contribution to the thermal resistivity, as treated above.

It is noteworthy that in the vicinity of the TQCP where the density of states $N(0)$ diverges, the number of collective modes associated with Eq. (2) and specified by two quantum numbers (orbital momentum $l$ and its projection $m$ ), becomes rather large. Accordingly, the task of finding the spectrum of these modes becomes quite complicated near the topological QCP, where many harmonics of the interaction function contribute to the dispersion equation (2). We address here the simple case $m=l$ in homogeneous matter where the dispersion equation reduces to [23]

$$
\frac{F_{l}}{(2 l) !} \int_{0}^{1}\left(P_{l}^{l}(x)\right)^{2} \frac{x^{2}}{s^{2}-x^{2}} d x=1,
$$

with $P_{l}^{l}(x)$ a spherical function and $F_{l}=f_{l} N(0)$. For a solution $s=c / v_{F}>1$ to exist, the harmonic $f_{l}$ must be positive. However, as seen from the case $l=1$ addressed above, although this requirement is necessary, it is not sufficient (recall that $s_{\min }=1$ ). In the present case, replacement of $x^{2} /\left(1-x^{2}\right)$ by $-1+1 /\left(1-x^{2}\right)$ allows both the integrals involved to be performed analytically, yielding

$$
\left(F_{l}\right)^{c r}=\left(f_{l}\right)^{c r} p_{F} M^{*} / \pi^{2}=2 l(2 l+1) .
$$

This result, setting the lower limit on the dimensionless parameter $F_{l}$ allowing propagation of the corresponding zero-sound mode, is in agreement with $\left(F_{1}\right)^{c r}=6$ in the zeron case $l=1$. As seen, the greater the orbital momentum $l$, the larger the effective mass $M^{*}$ should be to meet the condition involved. In this situation, the immediate vicinity of the TQCP tends to be overcrowded by different zero sounds, opening new boson channels that contribute weightily to the thermal conductivity $\kappa$.

Violation of WF law: flat-band/Peierls scenario. Returning to the case of $\mathrm{YbRh}_{2} \mathrm{Si}_{2}$, we observe that upon subtracting the posited zeron contribution to the thermal conductivity, the WF law breaks down on the disordered side of the $A F$ transition at $T_{N} \leq T<1 K$, the ratio $L(T) / L_{0}$ becoming less than unity [4, [5]. It is presumably significant that other instances of violation of the
WF law documented in the literature [8, 9] also involve a decline of the WF ratio.

This empirical conclusion is indicative of the failure of the standard FL scenario, in which nonzero values of $\rho_{0}$ and $w_{0}$ are attributed entirely to scattering of light carriers by impurities. Its inadequacy was revealed in measurements 29] of the electrical resistivity of the normal state of the superconducting heavy-fermion metal $\mathrm{CeCoIn}_{5}$ under pressure. These measurements showed a huge variation of the residual resistivity $\rho_{0}(P)$ in a narrow region of the $T-P$ phase diagram of this system near a critical pressure $P^{*}=1.6 \mathrm{GPa}$, even though the impurity number remained unchanged at any applied pressure.

In Ref. [30] such challenging behavior of $\mathrm{CeCoIn}_{5}$ was attributed to fermion condensation (FC). As indicated earlier, such a state arises from a rearrangement of the Landau state at a TQCP involving the formation of a flat band. A flat band is composed of the totality singleparticle states that belong to a portion of the spectrum that is completely flat at $T=0$, i.e., $\epsilon(\mathbf{p})=0$, and exhibits linear-in- $T$ dispersion at finite $T$ [16 20] (for recent reviews, see $31-34])$. Since quasiparticles in this state share the same energy, one has a situation analogous that of Bose condensation; hence the original name fermion condensate (FC) for the flat band. A remarkable feature of the FC/flat-band phenomenon is an inherent dramatic enhancement of the density of states, with a corresponding amplification of the superconducting critical temperature $T_{c}$ in systems hosting flat bands. This mechanism may in fact be relevant to the recent discovery of high- $T_{c}$ superconductivity in sulfur hydride [35].

Flat bands do provide a natural explanation of the striking behavior seen on the disordered side of the $\mathrm{AF}$ phase transition in the nonsuperfluid compound $\mathrm{YbRh}_{2} \mathrm{Si}_{2}$ [36 38], where the resistivity $\rho(T)$ shows linear-in- $T$ variation over an enormous temperature range from $T_{N}=70 \mathrm{mK}$ up to at least $7 \mathrm{~K}$. It must be noted that all the numerous versions of the standard critical-fluctuation Hertz-Millis approach fail to explain this pivotal observation.

With regard to the fate of the WF law in electron systems of solids possessing one or more flat bands, there exists a universal mechanism for its violation that is absent on the FL side of the underlying topological phase transition. This mechanism is associated with additional contributions to the conventional FL impurity-induced residual resistivities $\rho_{0}$ and $w_{0}$ that result from elastic scattering of light carriers by the heavy quasiparticles of flat bands, in a process analogous to elastic scattering of light carriers by impurities. In both cases, contributions to the damping $\gamma(T)$ of single-particle excitations are $T$ independent and proportional to the density of heavy carriers 30, 39.

Patently, if the two types of scattering were identical, the WF ratio in systems possessing flat bands would 
keep the same value as in FL theory. However, this is not the case, because the FC quasiparticles belong to the same electron system as the normal ones. It follows that in the case of a homogeneous charged Fermi liquid having flat bands but containing no impurities, for which the total electron momentum is necessarily conserved, the solution of the kinetic equation acquires a Peierls term $\delta n(\mathbf{p}) \propto \mathbf{p}$. This implies the presence of an analogous term proportional to momentum $\mathbf{p}$ in the electrical current $\mathbf{J}$ as well; accordingly, the electrical current flows without resistivity. As a result, in the actual case where the impurity-induced quantities $\rho_{0}$ and $w_{0}$ have nonzero values, $\rho_{0}$ remains unchanged through the fermion-condensation phase transition, while the thermal resistivity $w_{0}$, evaluated at $\mathbf{J}=0$, receives an addition contribution. This contribution, associated with the enhancement of the damping $\gamma(T)$, is proportional to the FC density. Thus, the WF ratio should be reduced from unity in charged Fermi liquids hosting flat bands.

The same situation prevails for electron systems of solids having flat bands. This is true despite the fact that the electron momentum is no longer conserved due to Umklapp processes, which provide for momentum transfer to the crystal lattice and lead to a nonzero residual resistivity $\rho_{0}$ even in the absence of impurities. The magnitude of $\rho_{0}$ then turns out to be proportional to the square of the Umklapp integral for $e-e$ collisions 25]. (Other processes, notably those involving collective modes, are ineffective in the limit $T \rightarrow 0$ relevant to the WF ratio.) The magnitude of the resulting contribution to $\rho_{0}$ turns out to be rather small, confirming the validity of the above prediction of a decline of the WF ratio in systems with the flat bands.

Let us now consider, within the flat-band scenario, how the WF ratio evolves under decrease of the impurity concentration. With improvement of purification efficiency, the influence of flat-band contributions to $\rho_{0}$ and $w_{0}$ continues to grow. Thus, starting at the critical level at which flat-band contributions become comparable to those from impurities, one expects to see a rapid enhancement of the decline of the WF ratio with further reduction of impurity concentration.

It is illuminating to apply this flat-band/Peierls scenario to assess the status of the WF law in electron systems subject to an external magnetic field $\mathbf{B}$. The essential point is that the Peierls contribution survives only through components of the current vector $\mathbf{J}$ parallel to the direction of the magnetic field $\mathbf{B}$. One has only to observe that this contribution is nullified if $\mathbf{J}$ is perpendicular to $\mathbf{B}$, since in this case the operators $\mathbf{J}$ and $\mathbf{B}$ do not commute with each other. Thus, even if the WF law is violated at $\mathbf{B}=\mathbf{0}$, imposition of a magnetic field, perpendicular to $\mathbf{J}$ leads to its recovery, in agreement with experiment [2, 7, 9].

Conclusions. We have proposed and explored two different scenarios, in some sense complementary, for viola- tion of the WF law in strongly correlated electron systems of solids. The implicated regions of the Lifshitz phase diagram lie in the neighborhood and on both sides of the TQCP at which the density of states diverges and the conventional Landau state loses its stability.

(i) Fermi-Liquid side of the topological quantum critical point (TQCP): zeron scenario. We have demonstrated that there emerges an additional channel of the thermal conductivity that is mediated by a new branch of collective excitations, a transverse zero-sound mode termed the zeron. This mechanism gives rise to an enhancement of the WF ratio $L(T) / L_{0}$ at finite temperatures on this side of the TQCP, while as $T$ goes down to 0 , so that $T<\Theta_{z}$, the WF ratio tends to 1 , and the WF law is recovered.

(ii) Non-Fermi-Liquid side of TQCP: flat-band/Peierls scenario. A quite different mechanism comes into play for those systems in which the topological transition leads to the formation of one or more flat bands featuring heavy quasiparticles. In this case an additional channel emerges in the electrical conductivity, associated with the occurrence of a Peierls term in the electrical current. This mechanism leads to a suppression of the Lorenz number $L(T)$ at sufficiently low temperatures, giving rise to the decline of the WF ratio $L(T \rightarrow 0) / L_{0}$. In the classical temperature region $T>\Theta_{D}$, this decline is eliminated, because elastic phonon contributions to both $\rho$ and $w$ prevail, so that the WF law is recovered.

The available experimental data [4, 5, , 7, 9] are consistent with the phenomenological analysis presented here.

Acknowledgements. The authors are grateful to J. Paglione and G. Volovik for valuable discussions. This research was partially supported by RFBR grants 13-0200085 and 15-02-06261, and by grant NS-932.2014.2 from the Russian Ministry of Sciences. VRS was supported by the Russian Science Foundation, Grant No. 14-22-00281. VAK thanks the McDonnell Center for the Space Sciences for timely support. JWC is indebted to the University of Madeira and its Centro de Ciências Matemáticas for gracious hospitality during a sabbatical residency.

[1] H. Pfau, S. Hartmann, U. Stockert et al., Nature 484, 493 (2012).

[2] Y. Machida, K. Tomokuni, K. Izawa et al., Phys. Rev. Lett. 110, 236402 (2014).

[3] J.-Ph. Reid. M. A. Tanatar, R. Daou et al., Phys. Rev. B89, 045130 (2014).

[4] M. Taupin, G. Knebel1, T. D. Matsuda et al., arXiv:1503.01991 (2015). 
[5] M. Taupin, G. Knebel1, T. D. Matsuda et al., Phys. Rev. Lett. 115, 046402 (2015).

[6] J.-Ph. Reid, Physics 8, 70 (2015).

[7] J. Paglione, M. A. Tanatar, D. G. Hawthorn et al., Phys. Rev. Lett. 97, 106606 (2006).

[8] M. A. Tanatar, J. Paglione, C. Petrovic, L. Taillefer, Science 316, 1320 (2007).

[9] J. K. Dong, Y. Tokiwa, S. L. Bud'ko et al., Phys. Rev. Lett. 110, 176402 (2013).

[10] P. Gegenwart, J. Custers, T. Tayama et al., Acta Phys. Pol. 34, 323 (2003).

[11] J. Custers P. Gegenwart, H. Wilhelm et al., Nature 424, 525 (2003).

[12] V. A. Khodel, J. W. Clark, V. R. Shaginyan, M. V. Zverev, JETP Lett. 92, 532 (2010).

[13] V. A. Khodel, J. W. Clark, M. V. Zverev, Physics of Atomic Nuclei 74, 1237 (2011).

[14] J. W. Clark, V. A. Khodel, and M. V. Zverev, Journ. Modern Phys. B27, 134005 (2013).

[15] V. A. Khodel, V. V. Khodel, V. R. Shaginyan, Phys. Rep. 249, 1, 1994.

[16] V. A. Khodel and V. R. Shaginyan, JETP Lett. 51, 553 (1990).

[17] G. E. Volovik, JETP Lett. 53, 222 (1991).

[18] P. Nozières, J. Phys. I France 2, 443 (1992).

[19] S.-S. Lee, Phys. Rev. D 79, 086006 (2009).

[20] D. Yudin, D. Hirschmeier, H. Hafermann et al., Phys. Rev. Lett. 112, 070403 (2014).

[21] L. D. Landau, Zh. Eksp. Teor. Fiz. 30, 1058 (1956); [Sov. Phys. JETP, 3, 920 (1957)].

[22] L. D. Landau, Zh. Eksp. Teor. Fiz. 35, 97 (1958); [Sov. Phys. JETP, 8, 70 (1958)].

[23] I. M. Khalatnikov, An Introduction to the Theory of Su- perfluidity (Perseus Publishing, Cambridge, 2000).

[24] A. B. Migdal, Theory of Finite Fermi Systems and Applications to Atomic Nuclei (Wiley, New York, 1967).

[25] L. D. Landau and E. M. Lifshitz, Physical Kinetics (Pergamon, Oxford, 1980).

[26] S. Hartmann, N. Oeschler, C. Krellner et al., Phys. Rev. Lett. 104, 096401 (2010).

[27] R. D. Barnard Thermoelectricity in Metals and Alloys (Taylor and Francis, London 1972).

[28] K. Behnia, D. Jaccard, and J. Flouquet, J. Phys.: Condens. Matter, 16, 5187 (2004).

[29] V. A. Sidorov, M. Nicklas, P. G. Pagliuso et al., Phys. Rev. Lett. 89, 157004 (2002).

[30] V. R. Shaginyan, A. Z. Msezane, K. G. Popov et al., Phys. Rev. B 86, 085417 (2012).

[31] J. W. Clark, M. V. Zverev, and V. A. Khodel, Annals of Physics, 327, 3063 (2012).

[32] M. Ya. Amusia, K. G. Popov, V. R. Shaginyan, V. A. Stephanovich, Theory of Heavy-Fermion Compounds, Springer Series in Solid-State Sciences 182, (2014).

[33] T. T. Heikkilä, G. E. Volovik, arXiv:1504.05824

[34] V. A. Khodel, Physics of Atomic Nuclei, 78, 20 (2015).

[35] A. P. Drozdov, M. I. Eremets, I. A. Troyan et al., Nature 525, 73 (2015).

[36] V. A. Khodel, V. R. Shaginyan, and P. Schuck, JETP Lett. 63, 752 (1996).

[37] V. A. Khodel, M. V. Zverev, Physica B 312-313, 506 (2002).

[38] V. A. Khodel, M. V. Zverev, J. W. Clark, JETP Lett. 81, 315 (2005).

[39] V. R. Shaginyan, A. Z. Msezane, K. G. Popov et al., Europhys. Lett. 106, 37001 (2014). 